\title{
Mimicking Physiological Cellular Environment of Protein Using Macromolecular Crowding Approach
}

\author{
Nasreen K and Islam $A^{*}$ \\ Centre for Interdisciplinary Research in Basic Sciences, India
}

*Corresponding author: Asimul Islam, Associate Professor, Member, National Academy of Sciences, India (NASI), Centre for Interdisciplinary Research in Basic Sciences, Jamia Millia Islamia, India, Tel: 0091-9312812007; Email: aislam@jmi.ac.in

\section{Review Article}

Volume 4 Issue 2

Received Date: September 07, 2020

Published Date: October 05, 2020

\section{Abstract}

The intracellular environment is highly crowded because of the presence of high concentration of biomolecules such as proteins, DNA, RNA, cytoskeleton etc and the concentration of these biomolecules in the cytoplasm is in the range of 80-400 $\mathrm{mg} \mathrm{ml}^{-1}$ which accounts for nearly 5-40 \% of the total cellular volume. These biomolecules generate a crowded medium having restricted amount of free water. Moreover, the volume occupied by co-solute is unavailable to other molecules, which results in the decrease of available volume to these molecules. Thus, macromolecular crowding can affect any reaction, which depends upon the available volume. The thermodynamic consequences of macromolecular crowding are known as excluded volume effects. Le Chatelier's principle leads to the conclusion that volume exclusion will always favor the folded state of the protein because this form is more compact and occupies lesser space than the denatured state. The volume exclusion phenomenon increases the entropy, which leads to decrease in the free energy of the system that leads to stabilization. Excluded volume shifts the equilibrium towards the native state because the free energy of the denatured states gets raised in crowded condition. It is also said that there is a direct effect of macromolecular crowding on the unfolded state because it increases the free energy of the denatured state and increases the relative stability of the folded state. Thus, macromolecular crowding forces the denatured state to become more compact. Thus it is expected that the behavior of any protein present in crowded environment is different from its behavior in the dilute solution. Thus, macromolecular crowding has made appreciable impact on protein structure, protein function, protein stability, protein folding, binding of ligand to protein, protein-protein interaction, molten globule formation and protein aggregation. Volume exclusion because of crowding agent increases the stability of protein and higher the volume exclusion greater is the stabilizing effect on protein. On the other hand, chemical interaction between crowder and protein overcomes the excluded volume effect leading to destabilization of the protein. Thus, the effect of crowder depends on the concentration, shape, size and nature of crowding agent employed.

Keywords: Macromolecular crowding; Protein stability; Volume exclusion

\section{Introduction}

Cell is the basic structural and functional unit of life. It is the smallest unit of life and is called the "building blocks of life". Cell consists of cytoplasm which is enclosed within the cell membrane and consists of enormous biomolecules such as nucleic acids, proteins, various metabolites and all the raw materials and machines required for protein synthesis and its folding. Mostly protein folding studies including characterization of their properties and behavior 
have been done in dilute buffer solution and it is assumed that this dilute condition also represents the in-vivo scenario. However, the cellular environment is entirely different from the dilute buffer condition [1-3]. Various biomolecules such as nucleic acids, carbohydrates, proteins, ribosomes etc. are present inside the cellular environment. Thus we can say that the intracellular environment is highly crowded due to the presence of these molecules. The nucleic acids, proteins and ribosomes have evolved to function in a crowded environment [4]. It is estimated that the concentration of macromolecules in the cytoplasm is in the range of $80-400 \mathrm{mg} / \mathrm{ml}$, which accounts for $5-40 \%$ of the total cellular volume $[1,3,5,6]$. This situation is defined as macromolecular crowding. The term "macromolecular crowding" was coined by Minton in 1981 [7] and it implies the influence of nonspecific steric repulsions caused by "inert" crowders on specific reaction that occurs in highly volume occupied medium. Presence of different macromolecules of different shapes, sizes and compositions govern the level of crowding inside cell [8]. For example, Escherichia coli cell has 300-400 $\mathrm{mg} \mathrm{ml}^{-1}$ biomolecules which consists of RNA, DNA and various proteins [3], human eye lens consists of $>500 \mathrm{mg} \mathrm{ml}^{-1}$ crystallin protein [9], RBC consists of $350 \mathrm{mg} \mathrm{ml}^{-1}$ hemoglobin [10] and the mitochondrial matrix consists of $500 \mathrm{mg} \mathrm{ml}^{-1}$ protein [11]. Crowding is not only confined to the cell interiors but also observed in the extracellular matrix. For example, blood plasma consists of macromolecules having a concentration of $80 \mathrm{mg} \mathrm{ml}^{-1}$, which can have significant crowding effect. Similarly cartilage also has enough crowding [8]. Crowding can have significant effect on structure, stability, functional activity, kinetics of protein folding and binding, protein aggregation and other complex biological processes [12]. Because the intracellular environment is highly crowded, it becomes very necessary and interesting how the proteins fold and function in the cellular environment. Understanding the effect of crowding is very important because the biophysical properties of proteins, process of protein folding and their interaction are usually obtained from studies in dilute environment where the effect of crowding is not taken into account. In order to study the effect of crowding on the biophysical properties of proteins, the crowded environment should be mimicked using either natural or synthetic crowding agents [13]. The cell extracts can be considered as the best macromolecular crowder because it can provide the actual cellular environment [8]. But because of various hurdles it becomes very difficult to perform and analyze experiments using the cell extract. Therefore, various natural and synthetic crowding agents are used in order to study the effect of crowding [12,14]. The cellular environment is composed of various biomolecules which differ in shape, size and nature [15]. Thus, crowders having different shape, size and nature were used in order to study the effect of crowding [12, 13,16-22]. The dependence of crowding effect on the concentration, size, shape and nature of crowding agents are very important attributes which must be considered [23]. It is very important to note that the crowder should be inert because steric repulsion is the only interaction between crowders and biomolecules. It results in decrease of available intracellular space to other macromolecules because of volume exclusion $[10,16]$.

\section{Protein Folding and Stability}

Folding of protein is a process in which an unstructured polypeptide chain folds into its native structure and thus attains a conformation, which is biologically functional. They are the most versatile molecule present in the living system and perform most of the essential biological functions. They are translated as nascent polypeptide chains with the help of ribosomes and various other proteins and mRNA. The polypeptide chains fold to their functionally active threedimensional structures [24]. The sequence of amino acid determines the particular three dimensional structures of proteins [25]. The sequence of amino acids and its threedimensional structure is responsible for its particular function. The ability of linear polypeptide to fold into its three dimensional structure is called as protein folding. The process of transcription and translation converts the genetic information into protein, which is the most significant molecule present in the cellular environment. Yet, how protein folding takes place and what structure a particular sequence of amino acids will generate is still not understood in detail. Therefore, the process of protein folding is often referred to as protein folding problem [26]. A sequence of amino acids folds into its native functionally active form within $10^{-3} \mathrm{sec}$ to $1 \mathrm{sec}$ [27]. Various forces required for protein folding are hydrophobic interactions, hydrogen bonding, van der Waals interactions and ionic interactions. These forces contribute to the structure, function and stability of proteins. Inside the cell, folding is a complex but regulated process having significant contribution of molecular chaperones as helper proteins. Indeed, the process of protein folding occurs when polypeptide chain on getting released from ribosomes enters the chaperones and gets released from there [28,29]. A nascent polypeptide chain does not encounter all the possible conformations during a finite time [30] and folding within a cell is controlled by chaperones. It is considered that the protein folding process is similar in in-vivo as well as in dilutes in-vitro condition, even if the two conditions are quite different [31,32]. It has been shown that the unfolded polypeptide can transform into unique and biologically active three dimensional structures in the dilute solution and without any contribution of helper protein [33]. Thus, a polypeptide has the ability to fold into unique three dimensional structures which depends only on the sequence of amino acids and present at suitable $\mathrm{pH}$, temperature and other environmental factors [33]. Anfinsen stated that protein folding is an energetically driven process where the native state has the minimum global free energy at 
that particular condition [33]. Proteins must fold and acquire a native three-dimensional structure in order to carry out its function. The native or folded form of any protein is more stable than the unfolded form. Thus increasing the stability of protein is very significant in basic protein research [34]. Proteins exist in dynamic equilibrium of $N$ (native) $\leftrightarrow D$ (denatured) state within the cellular environment [35-37]. Thus, the net balance of forces, which determine whether a protein will attain folded (native) conformation or unfolded confirmation is known as its stability. Stability indicates protein's resistance to adverse effects such as extreme $\mathrm{pH}$, temperature, pressure and chemical denaturants that affect the perseverance of its biological function or molecular integrity [38,39]. Unfolding of a protein loses the biological activity of it. Such unfolding is known as denaturation. For a reversible $\mathrm{N} \leftrightarrow \mathrm{D}$ process, the net thermodynamic stability of a protein is defined as the difference in Gibbs free energy between the native and the denatured states, i.e., $\Delta G_{\mathrm{D}}{ }^{\circ}=G_{\mathrm{D}}{ }^{\circ}$ $-G_{\mathrm{N}}^{\circ}$.

This difference in the free energies of native and denatured states are in the range of $5-15 \mathrm{kcal} \mathrm{mol}^{-1}$ which corresponds to a small number of weak non-covalent interactions [38]. The relative free energies of the native and the denatured states are the important factors, which influence its stability. The larger the value of $\Delta G_{\mathrm{D}}{ }^{\circ}$, the more is the stability of protein [40]. A subtle balance between the two thermodynamic quantities namely, change in enthalpy $[\Delta H(T)]$ and change in entropy $[\Delta S(T)]$ at a particular temperature contribute to protein stability. Moreover, the balance between several unfavorable and favorable interactions influences the protein folding and stability under physiological environments. Thus, stability provides a necessitated flexibility to protein so as to perform their functions in a proper manner [41].

\section{Thermodynamic Basis of Protein Stability}

A clear analysis of various thermodynamic properties such as entropy, enthalpy, and free energy is useful in understanding the protein stability. The classical works of Anfinsen [33,42] are the source for the concept of thermodynamic stability of proteins. The denatured state has substantial conformational freedom and high configurational entropy whereas the native state has low configurational entropy and is highly confirmationally restricted. In addition, the denatured state possesses large accessible surface area in comparison to the native state. Therefore, when a protein folds, a considerable loss in the entropy takes place. This loss must be equalized by a gain of enthalpy for the free energy to favor the process of folding. The enthalpy of side chains packing in the native state is favorable and recompenses for its low entropy. It is not just the enthalpy and entropy of the native and denatured states that contribute to the thermodynamic properties of a protein but the enthalpy and entropy of water must also be counted. Thus, the thermodynamic stability of protein is not only dependent on the intermolecular and intramolecular interactions but on the enthalpy and entropy of both the polypeptide chain and solvent molecules surrounding the protein [43].

The hydrophobic side chains of the native state of a protein are packed in hydrophobic cores and thus shielded from water. On the other hand, the hydrophobic side chains of the denatured state are exposed to solvent. Furthermore, the water molecules stack around these side chains, since they increase their hydrogen bonds with one another. This results in lowering of the entropy of water because the individual molecules possess less freedom of movement, and decrease in the enthalpy as more hydrogen bonds are generated [44]. In the same way, the donors and acceptors of hydrogen bond in the polypeptide backbone of the denatured protein are largely exposed to solvent and hence, bind more water molecules [45]. Consequently, these water molecules are released when the protein folds, and the increase in the entropy of water balances significantly for the loss of conformational entropy.

The atoms of the side-chains are closely packed together in the inner core of the folded protein. This close packing of amino acids is due to the contribution of the non covalent interactions such as hydrogen bonding, ionic bonds, van der Waals, and dipole-induce dipole forces and the favorable interactions between the groups within the native state of protein give rise to enthalpic effects [41]. The favorable enthalpy change $(\Delta H<0)$, which is associated with protein folding is indeed dependent on these favorable interactions. Hydrogen bonding and ionic interactions have significant roles in the stability of the native state conformation; however, their contribution to the free energy change is very less. Since, all possible hydrogen bond donors and acceptors form hydrogen bonds in a protein, whether they are in the native or denatured states of protein [46]. In the native state, intramolecular hydrogen bonding is dominant that leads to stabilization of secondary structural elements, whereas in the denatured state, mostly intermolecular hydrogen bonding takes place, i.e., hydrogen bond formation between amino acids residues and surrounding water molecule. The formation of intramolecular hydrogen bondings also takes place between the buried polar groups [47-53]. There is a little difference between the energy of H-bonds formation, between a $\mathrm{C}=\mathrm{O}$ and $\mathrm{H}-\mathrm{N}$ or between a $\mathrm{C}=\mathrm{O}$ and water. Thus, if intermolecular hydrogen bonding is lost, and intramolecular hydrogen bonding is reformed, the change in enthalpy component would be zero. Nevertheless, if the interaction between the polar groups of protein and water is lost and if the intramolecular hydrogen bonding does not occur, there will be unfavorable change in enthalpy. Consequently, 
in order to stabilize the folded state, protein considerably undergoes the formation of intramolecular hydrogen bonds between the polar groups within the protein so as to make it enthalpically favorable. Additionally, for the backbone groups of protein, the formation of secondary structural components efficiently takes control over this problem. Thus, for the correctly folded (native) conformation, formation of specific hydrogen bonds is essential.

\section{Macromolecular Crowding}

Most of the times, it is assumed that folding process and structural and biophysical properties of an isolated protein observed in dilute solutions are similar to those when it is present in the actual cellular environment. However, because of the presence of huge quantity of insoluble and soluble biomolecules, which includes nucleic acid, proteins, osmolytes, carbohydrates and ribosomes, the intracellular environment, is highly crowded $[8,54]$. There is an estimate that the macromolecular concentration in the cytoplasm ranges between 80 and $400 \mathrm{mg} / \mathrm{ml}$ [6]. All macromolecules present in cell interior collectively occupy $10-40 \%$ of the total cell volume [5]. This implies that a large portion of the intracellular space is unavailable to other macromolecules. The term macromolecular crowding which has been coined by Minton [55], implies the influence of steric repulsions on particular reaction that takes place in highly crowded environment.

In the late 1950's, the impact of macromolecular crowding have been identified, where investigation on the distribution of available holes for a spherical object in a network of fibres was presented through computing the possibility of inserting a particle of radius ' $r$ ' in any point within the network [56]. Subsequently, utilizing this method with a few alterations on the way the fibres were handled, it was found that addition of a crowding agent to the solution increases the chemical potential, an outcome that could be rationalized by a simple steric theory [57]. But then Minton and Wilf [55] presented the term "macromolecular crowding effect" to describe the effect of a crowded condition in biological processes. Afterwards, many researchers initiated to observe and examine the properties of proteins and other biomolecules in the milieus that may bear a resemblance to those present inside the cell. The phenomenon of macromolecular crowding implies the presence of nonspecific interactions (steric repulsion) of molecules. The universality of this phenomenon in biological solutions has been related to that of gravity, believing the fact that the living organisms have to deal with its outcomes [58]. Assessing the consequences of this effect at the molecular level could result in a deeper recognition of the physical principles that administrate the molecular interactions in cells. The nonspecific steric repulsion exists as the most essential of all interactions between the molecules in solution.
Therefore, it is more precisely termed as the excluded volume effect or volume exclusion when at the same instant of time, not a portion of any two macromolecules can be at the same position, i.e., there exists the mutual inapproachability of the molecules in a solution $[10,16,59]$. Therefore, that fraction of the total volume, which cannot be inhabited by the center of mass of a specific molecule at particular instant of time is known as the excluded volume, and the portion of the total volume that can be occupied is known as the available volume. While the part of the volume which is occupied by macromolecules of certain size enhances, there is a rapid decrease in the fraction of volume which is available to other macromolecule, and hence becomes considerably less than the volume fraction which is available to solvent i.e., water [58]. By placing shells around the background molecules of size is equal to the radius of the test molecule, the available volume can be envisaged appropriately [60].

Thus, many large molecules in a cell are present in such a way that a considerable portion of the intracellular volume is not available to other macromolecules, thereby leading to reduction in the available volume [61-63]. Owing to volume exclusion, any reaction that resulted in the augmentation of the available volume is enthused by macromolecular crowding $[16,64,65]$ as demonstrated in the thermodynamic analysis of multi component systems [66]. Hence, this macromolecular crowding phenomenon, implies the influence of excluded volume upon the processes that occur in crowded or immensely volume occupied medium [62].

\section{Outcome of Macromolecular Crowding}

Crowding results in volume exclusion, specific as well as non-specific intermolecular interactions and increase in viscosity. The various non-specific intermolecular interactions include weak interaction such as hydrogen bonding, van der Waals forces and weak ionic interactions etc. Increase in viscosity does not affect thermodynamic properties of the protein except when the system contains a hydrophobic-hydrophilic interface [67]. However, volume exclusion and intermolecular interactions will affect protein thermodynamic properties by shifting the folding equilibrium [68]. The areas where macromolecular crowding has made significant effect are protein function, structure and stability [69]. Theoretical and experimental studies have shown significant effects of macromolecular crowding on the kinetics and thermodynamics of many biomolecules $[67,70$ 72]. The influence of crowding on the stability of protein is believed to arise due to two phenomena, namely, chemical interactions and hard-core repulsion. The hard-core repulsion is always thought to be stabilizing as it involves only the molecular arrangements, which stabilize proteins by increasing the entropy of the system. Molecular interaction is thought to act enthalpically. The weak molecular interaction 
is also termed as soft interaction. The molecular interaction is thought to be destabilizing in nature.

\section{Volume Exclusion: Theoretical Models}

The aim of our study is to investigate the consequences of excluded volume effects on the proteins resulting from steric repulsion. Due to the presence of large number of macromolecules inside the cell, the effect of volume exclusion is particularly significant in vivo and it takes place with all macromolecules. The polymer chemist Kuhn was the first to propose the concept of volume exclusion in order to explicate the observation that less compaction was shown by real polymer chains in the absence of excluded volume effects than expected [73]. The explanation of nonideal gases (employing the van't Hoff isobar) also depends upon the concept of volume exclusion [74], which simply says that two molecules cannot occupy the same place at the same instant of time. The species which are nonspherical can exhibit much larger excluded volumes than the spherical species of the same size [7,75]. MacMillan \& Mayer investigated the related phenomenon by conducting a theoretical modeling study testing the real fluids [76]. They computed the amount of work needed to place one new particle into a fluid comprising a large number of other particles. They showed that their solution depends on viral coefficients and a certain interaction function between the molecules. The principle constraint is that only hard core repulsion has been used, i.e., it was assumed that the only crowding effect is the infinite potential if the overlap distance is more than distance between two particles. This constraint has an advantage of modeling the whole set of possible molecular interactions by demonstrating each particle as a single object with a specified size and shape. Though, in general, it is essential to acclimatize this approach to systems comprising of various types of molecules. To deal with this issue, the most common mode includes the application of approximate models which are based on scaled particle theory (SPT) [77]. Initially, this approach was developed to depict the alterations in the activity for fluids containing hard sphere particles [78], however, later it was expanded to mask non-spherical particles also $[79,80]$. SPT is specifically useful in order to develop theoretical understandings into the influence of macromolecular crowding on the properties of protein. Since proteins are polymers, the notion of volume exclusion can include the proteins present in the solutions consisting of large numbers of several other proteins. Laurent and Ogston [81,82] developed one of the first theories of this category where they learned about size exclusion chromatography after initial analysis of the effects of hyaluronic acid at large concentrations on the partitioning of protein by Ogston [56,57]. In their study, the protein was supposed to be a sphere and the dextran chromatographic matrix was considered as an array of rods through which the proteins have to migrate. Subsequently, using a hard sphere approximation, Minton developed a model of protein activity [83]. This approximation was established on the basis of dependence of osmotic pressure for concentrated solutions of hemoglobin, which were presumed to act like clusters of hard spheres that were supposed to interact with one another only by means of hard core steric repulsion [83-85]. Afterwards, Minton described protein folding and unfolding processes and protein association equilibria in the context of his model, where he considered both the folded and unfolded states of a protein as approximate hard spheres [2]. Consequently, the average extension and the representation of the unfolded state as the hard sphere particle displays greater volume occupation than the compact folded state. Thus, Minton's hard sphere model with SPT predicted the consequence of inert and large background species on protein folding equilibria $[2,75]$. It was predicted that the process of folding in the presence of background molecules of spherical shape bring about a non-linear rise in its equilibrium constant. Furthermore, in order to estimate the outcomes of crowding on protein folding equilibria, an analogous model was applied to non-spherical objects [75]. Predictions were made by other researchers also using similar approaches as Minton's but those predictions were based on distinct assumptions for the unfolded state. For instance, a Gaussian chain model was applied by Zhou for the unfolded state in the presence of crowders of spherical shape, while representing the folded state as a hard sphere particle [86]. A conversion from stabilization to destabilization of the folded state was predicted in the presence of high concentrations crowders using this model, since the Gaussian chain provide accommodations for voids between the background species [87-88]. This resulted in a weak destabilization of the unfolded state in comparison to the folded state. Consequently, Minton proposed an analogous model in which the unfolded state was assumed to act like a Gaussian cloud. It was predicted that the enlargement of the unfolded ensemble decreases, however, no noticeable change in the overall stabilization effect was monitored comparative to that obtained employing the previous hard-sphere model [64]. Thus, all of these models share a similarity that they assume the folded state as a hard sphere, while their representation of the unfolded state is different.

It is argued that crowder stabilizes the native state of proteins indirectly by compacting the denatured state that is more extended and flexible [70,87]. Macromolecular crowding has also shown its effect by compressing the structure of nucleic acids [88]. Hence macromolecular crowding destabilizes the unfolded protein entropically which shifts the equilibrium towards the folded state, which leads to increase in stability. Le Chatelier's principle leads us to conclude that exclusion of volume will always favor the native state of protein because it occupies lesser space. 
Physical Science \& Biophysics Journal

\section{Chemical Interaction}

Contrary to the above observations, a report shows that protein when used as a crowder can destabilize another protein [89]. Furthermore, different crowding agents may have different effects on the same protein, e.g., stability of apo-HLA is increased significantly by dextran 70 and moderately by ficoll 70 while PEG significantly decreases its stability [90]. There has been report that PEG binds to human serum albumin resulting in decrease of its $\alpha$-helical content while increasing the $\beta$-turn content [91], and presence of PEG results in loss of secondary structure of thiol protease [92]. It has also been observed that PEG interacts with lysozyme, ribonuclease $\mathrm{A}$, lactoglobulin, bovine serum albumin and chymotrypsinogen A resulting in their destabilization [93]. Crowders such as PEG and ficoll 70 destabilize ubiquitin and myoglobin $[69,94]$. It is not that PEG always destabilizes proteins; it has been observed that PEG can stabilize protein such as $\alpha$-chymotrypsin [95]. The mechanism of action of synthetic crowders has been disagreed owing to a few studies, which implied that synthetic crowders such as dextran, ficoll 70 and polyvinyl pyrrolidone showed combined effect of steric and molecular interaction on proteins such as CI2, ubiquitin and SOD [96-98]. Molecular or enthalpic interactions can be unfavorable or favorable towards protein stability. If the interaction is non-specific, it will dominate in the unfolded state of protein and thus destabilize it. As a result interaction will counteract the "excluded volume effect" which results in no or even destabilizing effect on protein stability [16]. It is very obvious that soft interactions are always present in the cell interior, since proteins have several exposed charged residues. In fact, interactions between proteins are present to assist an extracellular level of organization [99], however, these soft interactions are often ignored when protein folding is studied in the light of macromolecular crowding [100].

\section{References}

1. Fulton AB (1982) How crowded is the cytoplasm? 30(2): 345-347.

2. Minton AP (1983) The effect of volume occupacy upon the thermodynamic activity of proteins: some biochemical consequences. Mol Cell Biochem 55: 119-140.

3. Zimmerman SB, Trach SO (1991) Estimation of macromolecule concentrations and excluded volume effects for the cytoplasm of Escherichia coli. J Mol Biol 222(3): 599-620.

4. Medalia O, Weber I, Frangakis AS, Nicastro D, Gerisch G, et al. (2002) Macromolecular architecture in eukaryotic cells visualized by cryoelectron tomography. Science 298(5596): 1209-1213.

5. Ellis RJ, Minton AP (2003) Cell biology: join the crowd.
Nature 425(6953): 27-28.

6. Rivas G, Ferrone F, Herzfeld J (2004) Life in a crowded world, EMBO Reports 5(1): 23-27.

7. Minton AP (1981) Excluded volume as a determinant of macromolecular structure and reactivity, Biopolymers 20(10): 2093-2120.

8. Ellis RJ (2001) Macromolecular crowding: obvious but underappreciated, Trends Biochem Sci 26(10): 597-604.

9. Harding JJ (1991) Cataract: Biochemistry, Epidemiology and Pharmacology. $1^{\text {st }}($ Edn.), Springer Netherlands, Chapman and Hall, London pp: 334.

10. Ellis RJ (2001) Macromolecular crowding: an important but neglected aspect of the intracellular environment. Curr Opin Struct Biol 11(1): 114-119.

11. Srere PA (1980) The infrastructure of the mitochondrial matrix. Trends in Biochemical Sciences 5(5): 120-121.

12. Zhou HX, Rivas G, Minton AP (2008) Macromolecular crowding and confinement: biochemical, biophysical, and potential physiological consequences. Annu Rev Biophys 37: 375-397.

13. Chebotareva NA, Kurganov BI, Livanova NB (2004) Biochemical effects of molecular crowding. Biochemistry 69: 1239-1251.

14. Jiang M, Guo Z (2007) Effects of Macromolecular Crowding on the Intrinsic Catalytic Efficiency and Structure of Enterobactin-Specific Isochorismate Synthase. Journal of the American Chemical Society 129(4): 730-731.

15. Goodsell DS (1991) Inside a living cell. Trends in Biochemical Sciences 16: 203-206.

16. Zimmerman SB, Minton AP (1993) Macromolecular crowding: biochemical, biophysical, and physiological consequences, Annu Rev Biophys Biomol Struct 22: 2765.

17. Minton AP (2001) The Influence of Macromolecular Crowding and Macromolecular Confinement on Biochemical Reactions in Physiological Media. Journal of Biological Chemistry 276(14): 10577-10580.

18. Samiotakis A, Wittung-Stafshede P, Cheung MS (2009) Folding, stability and shape of proteins in crowded environments: experimental and computational approaches. Int J Mol Sci 10(2): 572-588.

19. Christiansen A, Wang Q, Cheung MS, Wittung-Stafshede $P$ (2013) Effects of macromolecular crowding agents on protein folding in vitro and in silico. Biophysical Reviews 


\section{Physical Science \& Biophysics Journal}

5(2): 137-145.

20. Kuznetsova IM, Turoverov KK, Uversky VN (2014) What Macromolecular Crowding Can Do to a Protein. International Journal of Molecular Sciences 15(12): 23090-23140.

21. Kuznetsova IM, Zaslavsky BY, Breydo L, Turoverov KK, Uversky VN (2015) Beyond the excluded volume effects: mechanistic complexity of the crowded milieu. Molecules 20(1): 1377-1409.

22. Pastor I, Vilaseca E, Madurga S, Garces JL, Cascante M, et al. (2011) Effect of crowding by dextrans on the hydrolysis of N-Succinyl-L-phenyl-Ala-p-nitroanilide catalyzed by alpha-chymotrypsin. J Phys Chem B 115(5): 1115-1121.

23. Batra J, Xu K, Zhou HX (2009) Nonadditive effects of mixed crowding on protein stability. Proteins $77(1)$ : 133-138.

24. Roman MB (2012) Macromolecular crowding effects in the mechanical unfolding forces of proteins. Drexel University, USA.

25. Socolich M, Lockless SW, Russ WP, Lee H, Gardner KH, et al. (2005) Evolutionary information for specifying a protein fold. Nature 437(7058): 512-518.

26. Creighton TE (1990) Protein folding. Biochemical Journal 270(1): 1-16.

27. Creighton TE (1993) Proteins: Structure and molecular properties. $2^{\text {nd }}(E d n)$, Freeman, WH \& Co., New York, USA, pp: 244-249.

28. Ellis RJ (1994) Roles of molecular chaperones in protein folding. Current Opinion in Structural Biology 4(1): 117 122.

29. Weissman JS, Rye HS, Fenton WA, Beechem JM, Horwich AL (1996) Characterization of the active intermediate of a GroEL-GroES-mediated protein folding reaction. Cell 84(3): 481-490.

30. Levinthal C (1968) Are there pathways for protein folding?, J Chim Phys 65: 44-45.

31. Doig AJ, Williams DH (1991) Is the hydrophobic effect stabilizing or destabilizing in proteins? The contribution of disulphide bonds to protein stability. J Mol Biol 217(2): 389-398.

32. Cooper A, Eyles SJ, Radford SE, Dobson CM (1992) Thermodynamic consequences of the removal of a disulphide bridge from hen lysozyme. J Mol Biol 225(4): 939-943.
33. Anfinsen CB (1973) Principles that govern the folding of protein chains. Science 181(4096): 223-230.

34. Pace CN, Grimsley GR (2001) Protein Stability. John Wiley \& Sons, Ltd, USA.

35. Tanford C (1970) Protein denaturation. Part C. Theoretical models for the mechanism of denaturation. Adv Protein Chem 24: 1-95.

36. Tanford C (1968) Protein denaturation. Adv Protein Chem 23: 121-282.

37. Pace CN (1975) The stability of globular proteins. CRC Crit Rev Biochem 3(1): 1-43.

38. Jaenicke R (1991) Protein stability and molecular adaptation to extreme conditons. European Journal of Biochemistry 202(3): 715-728.

39. Jaenicke R, Bohm G (1998) The stability of proteins in extreme environments. Curr Opin Struct Biol 8(6): 738748.

40. Becktel WJ, Schellman JA (1987) Protein stability curves. Biopolymers 26(11): 1859-1877.

41. Kauzmann W (1959) Some factors in the interpretation of protein denaturation. Adv Protein Chem 14: 1-63.

42. Anfinsen CB (1956) The limited digestion of ribonuclease with pepsin. J Biol Chem 221(1): 405-412.

43. Makhatadze GI, Privalov PL (1995) Energetics of Protein Structure. Advances in Protein Chemistry 47: 307-425.

44. Privalov PL, Makhatadze GI (1993) Contribution of hydration to protein folding thermodynamics. II. The entropy and Gibbs energy of hydration. J Mol Biol 232(2): 660-679.

45. Makhatadze GI, Privalov PL (1996) On the entropy of protein folding. Protein Science 5(3): 507-510.

46. McDonald IK, Thornton JM (1994) Satisfying hydrogen bonding potential in proteins. J Mol Biol 238(5): 777793.

47. Schmid FX (2001) Biological Macromolecules: UV-visible Spectrophotometry. John Wiley \& Sons, Ltd, USA.

48. Kendrew JC, Bodo G, Dintzis HM, Parrish RG, Wyckoff $\mathrm{H}$, et al. (1958) A Three-Dimensional Model of the Myoglobin Molecule Obtained by X-Ray Analysis. Nature 181: 662-666.

49. Christiansen A (2013) Effects of Macromolecular Crowding on Protein Folding. Umeå universitet, Umeå, pp: 96.

50. Chen YH, Yang JT, Chau KH (1974) Determination of the 
helix and beta form of proteins in aqueous solution by circular dichroism. Biochemistry 13(16): 3350-3359.

51. Chen YH, Yang JT, Martinez HM (1972) Determination of the secondary structures of proteins by circular dichroism and optical rotatory dispersion. Biochemistry 11(22): 4120-4131.

52. Mackenzie RC (1975) Thermal Analysis. In: Nicol AW (Ed.), Physicochemical Methods of Mineral Analysis. Springer US, Boston, USA, pp: 389-420.

53. Watson ES, O’Neill MJ, Justin J, Brenner N (1964) A Differential Scanning Calorimeter for Quantitative Differential Thermal Analysis. Analytical Chemistry 36(7): 1233-1238.

54. Stagg L, Zhang SQ Cheung MS, Wittung-Stafshede P (2007) Molecular crowding enhances native structure and stability of alpha/beta protein flavodoxin. Proc Natl Acad Sci USA 104(48): 18976-18981.

55. Minton AP, Wilf J (1981) Effect of macromolecular crowding upon the structure and function of an enzyme: glyceraldehyde-3-phosphate dehydrogenase. Biochemistry 20(17): 4821-4826.

56. Ogston AG (1958) The spaces in a uniform random suspension of fibres. Transactions of the Faraday Society 54: 1754-1757.

57. Ogston AG, Phelps CF (1961) The partition of solutes between buffer solutions and solutions containing hyaluronic acid. Biochemical J 78(4): 827-833.

58. Minton AP (2006) Macromolecular crowding. Current Biology 16(8): R269-R271.

59. Minton AP (2001) The influence of macromolecular crowding and macromolecular confinement on biochemical reactions in physiological media. J Biol Chem 276(14): 10577-10580.

60. Fulton A (1982) How crowded is the cytoplasm ?, Cell $30(2): 345-347$.

61. Christiansen A (2013) Effects of Macromolecular Crowding on Protein Folding. Umea university.

62. Minton AP (2006) How can biochemical reactions within cells differ from those in test tubes? Journal of Cell Science 119: 2863-2869.

63. Ralston GB (1990) Effects of "crowding" in protein solutions. J Chem Educ 67(10): 857.

64. Minton AP (2005) Models for Excluded Volume Interaction between an Unfolded Protein and Rigid Macromolecular Cosolutes: Macromolecular Crowding and Protein Stability Revisited, Biophysical Journal 88(2): 971-985.

65. Zhou HX (2004) Protein folding and binding in confined spaces and in crowded solutions, Journal of Molecular Recognition 17(5): 368-375.

66. Eisenberg H (1990) Thermodynamics and the structure of biological macromolecules. Eur J Biochem 187: 7-22.

67. Beg I, Minton AP, Islam A, Hassan MI, Ahmad F (2017) The $\mathrm{pH}$ Dependence of Saccharides' Influence on Thermal Denaturation of Two Model Proteins Supports an Excluded Volume Model for Stabilization Generalized to Allow for Intramolecular Electrostatic Interactions. J Biol Chem 292: 505-511.

68. Christiansen A, Wittung-Stafshede P (2014) Synthetic crowding agent dextran causes excluded volume interactions exclusively to tracer protein apoazurin. FEBS Lett 588(5): 811-814.

69. Malik A, Kundu J, Mukherjee SK, Chowdhury PK (2012) Myoglobin Unfolding in Crowding and Confinement. The Journal of Physical Chemistry B 116(43): 12895-12904.

70. Cheung MS, Klimov D, Thirumalai D (2005) Molecular crowding enhances native state stability and refolding rates of globular proteins. Proceedings of the National Academy of Sciences of the United States of America 102(13): 4753-4758.

71. Shahid S, Ahmad F, Hassan MI, Islam A (2015) Relationship between protein stability and functional activity in the presence of macromolecular crowding agents alone and in mixture: An insight into stabilityactivity trade-off. Arch Biochem Biophys 584: 42-50.

72. Beg I, Minton AP, Hassan I, Islam A, Ahmad F (2015) Thermal Stabilization of Proteins by Mono- and Oligosaccharides: Measurement and Analysis in the Context of an Excluded Volume Model. Biochemistry 54(23): 3594-3603.

73. Kuhn W (1934) Über die Gestalt fadenförmiger Moleküle in Lösungen. Kolloid-Zeitschrift 68: 215.

74. Wedler G (2004) Textbook of physical chemistry. $5^{\text {th }}$ (Edn.), Wiley-VCH Verlag GmbH \& Co, USA.

75. Minton AP (1998) Molecular crowding: analysis of effects of high concentrations of inert cosolutes on biochemical equilibria and rates in terms of volume exclusion. Methods Enzymol 295: 127-149.

76. McMillian WG, Mayer JE (1945) The Statistical Thermodynamics of Multicomponent Systems. J Chem Phys 13: 276-305. 
77. Poddar NK, Ansari ZA, Singh RK, Moosavi Movahedi AA, Ahmad F (2010) Effect of oligosaccharides and their monosaccharide mixtures on the stability of proteins: a scaled particle study. J Biomol Struct Dyn 28(3): 331341.

78. Reiss H, Frisch HL, Lebowitz JL (1959) Statistical Mechanics of Rigid Spheres. J Chem Phys 31: 369-380.

79. Boublík T (1974) Statistical thermodynamics of convex molecule fluids. Molecular Physics 27: 1415-1427.

80. Gibbons RM (1969) The scaled particle theory for particles of arbitrary shape. Molecular Physics 17(1): 81-86.

81. Ogston AG, Silpananta P (1970) The thermodynamics of interaction between Sephadex and penetrating solutes. Biochemical Journal 116(2): 171-175.

82. Ogston AG (1970) On the interaction of solute molecules with porous networks. The Journal of Physical Chemistry 74(3): 668-669.

83. Ross PD, Minton AP (1977) Analysis of non-ideal behavior in concentrated hemoglobin solutions. Journal of Molecular Biology 112(3): 437-452.

84. Ross PD, Minton AP (1979) The effect of non-aggregating proteins upon the gelation of sickle cell hemoglobin: Model calculations and data analysis. Biochemical and Biophysical Research Communications 88(4): 13081314.

85. Guttman HJ, Anderson CF, Record MT (1995) Analyses of thermodynamic data for concentrated hemoglobin solutions using scaled particle theory: implications for a simple two-state model of water in thermodynamic analyses of crowding in vitro and in vivo. Biophysical Journal 68(3): 835-846.

86. Zhou HX (2008) Protein folding in confined and crowded environments. Arch Biochem Biophys 469(1): 76-82.

87. Zhou HX (2004) Loops, Linkages, Rings, Catenanes, Cages, and Crowders: Entropy-Based Strategies for Stabilizing Proteins. Acc Chem Res 37(2): 123-130.

88. Harve KS, Lareu R, Rajagopalan R, Raghunath M (2010) Understanding how the crowded interior of cells stabilizes DNA/DNA and DNA/RNA hybrids-in silico predictions and in vitro evidence. Nucleic Acids Res 38(1): 172-181.

89. Schlesinger AP, Wang Y, Tadeo X, Millet O, Pielak GJ
(2011) Macromolecular Crowding Fails To Fold a Globular Protein in Cells. Journal of the American Chemical Society 133(21): 8082-8085.

90. Zhang DL, Wu LJ, Chen J, Liang Y (2012) Effects of macromolecular crowding on the structural stability of human alpha-lactalbumin. Acta Biochim Biophys Sin 44(8): 703-711.

91. Ragi C, Sedaghat-Herati MR, Ouameur AA, TajmirRiahi HA (2005) The effects of poly(ethylene glycol) on the solution structure of human serum albumin. Biopolymers 78(5): 231-236.

92. Fatima S, Khan RH (2007) Effect of polyethylene glycols on the function and structure of thiol proteases. J Biochem 142(1): 65-72.

93. Lee JC, Lee LL (1981) Preferential solvent interactions between proteins and polyethylene glycols. J Biol Chem 256(2): 625-631.

94. Senske M, Törk L, Born B, Havenith M, Herrmann C, et al. (2014) Protein Stabilization by Macromolecular Crowding through Enthalpy Rather Than Entropy. Journal of the American Chemical Society 136(25): 9036-9041.

95. Simon LM, Kotorman M, Szabo A, Garab G, Laczko I (2004) Effects of polyethylene glycol on stability of alpha-chymotrypsin in aqueous ethanol solvent. Biochem Biophys Res Commun 317(2): 610-613.

96. Benton LA, Smith AE, Young GB, Pielak GJ (2012) Unexpected effects of macromolecular crowding on protein stability. Biochemistry 51(49): 9773-9775.

97. Wang Y, Sarkar M, Smith AE, Krois AS, Pielak GJ (2012) Macromolecular crowding and protein stability. J Am Chem Soc 134(40): 16614-16618.

98. Jiao M, Li HT, Chen J, Minton AP, Liang Y (2010) Attractive Protein-Polymer Interactions Markedly Alter the Effect of Macromolecular Crowding on Protein Association Equilibria. Biophys J 99(3): 914-923.

99. Wirth AJ, Gruebele M (2013) Quinary protein structure and the consequences of crowding in living cells: leaving the test-tube behind. Bioessays 35(11): 984-993.

100. Luby-Phelps K, Castle PE, Taylor DL, Lanni F (1987) Hindered diffusion of inert tracer particles in the cytoplasm of mouse 3T3 cells. Proc Natl Acad Sci USA 84(14): 4910-4913. 